\title{
Un nouveau Microcharon (Isopoda, Janiroidea) du Maroc - origine et biogéographie historique
}

\author{
M. Boulanouar ${ }^{1}$, M. Yacoubi ${ }^{1}$, M. Messouli ${ }^{1} \&$ N. Coineau ${ }^{2}$ \\ ${ }^{1}$ Université de Marrakech, Laboratoire d'Hydrobiologie et Ecologie, Faculté des Sciences Semlalia, \\ B.P. S 15, Marrakech, Maroc; ${ }^{2}$ Université P. et M. Curie, U.R.A. C.N.R.S. 117, Observatoire \\ Océanologique de Banyuls, Laboratoire Arago, 66650 Banyuls-sur-Mer, France
}

Keywords: Taxonomy, Crustacea, Isopoda, interstitial stygobionts, Microcharon, Morocco, historical biogeography

\begin{abstract}
The interstitial crustacean isopods of the genus Microcharon (Microparasellidae) are relatively well-diversified and widely distributed in Morocco. A new species, M. boutini, is described. The 5-dentate mandibular pars incisiva, the elongated inner lobe of the exopodite, and the reduced armature of the first male pleopod characterize the species. $M$. boutini $\mathrm{n}$. sp. belongs to the monophyletic messoulii group of species, the most primitive representatives of the genus. The distribution of $M$. boutini n. sp. covers both the northwestern part of the Jbilet and the nearest adjacent plain, as well as the High Atlas of the Marrakech piedmont. From a historical biogeographic point of view, interstitial ancestral marine populations would have been left in subterranean continental waters by the regression of the "Phosphate Sea" during the Lutetian (Eocene). The distribution of the same species both in High Atlas valleys and in the Jbilet region poses an evolutionary rate problem.
\end{abstract}

\section{Résumé}

Les Crustacés Isopodes interstitiels du genre Microcharon (Microparasellidae) sont relativement bien diversifiés et largement répandus au Maroc. Une espèce nouvelle, $M$. boutini, est décrite. La pars incisiva de la mandibule à 5 dents et le pléopode 1 du mâle au lobe interne de l'exopodite allongé et au nombre de soies incomplet caractérisent bien l'espèce. Elle appartient au groupe monophylétique d'espèces messoulii, les plus primitives du genre. La répartition de $M$. boutini $n$. sp. englobe le nordouest du massif des Jbilet et la plaine en contre-bas, ainsi que le piémont du Haut-Atlas de Marrakech. Du point de vue de la biogéographie historique, les populations marines ancestrales interstitielles ont dû pénétrer dans les eaux souterraines continentales lors de la régression de la "Mer des Phosphates", au Lutétien, pendant l'Eocène. $M$. boutini dérive donc d'un ancêtre stygobie lutétien. La présence de la même espèce dans deux vallées du Haut-Atlas et dans la région des Jbilet pose le problème de la vitesse de l'évolution.

\section{Introduction}

Les Isopodes stygobies constituent une composante non négligeable des peuplements interstitiels du Maroc (Boulanouar, 1986; Boutin \& Boulanouar, 1984; Messouli, 1984; Boulal, 1988; Idbennacer, 1990; Yacoubi, 1990). Les Cirolanides paraissent les plus diversifiés et sont distribués dans de nombreuses régions (Nourisson, 1956; Botosaneanu et al., 1985; Boutin \& Boulanouar, 1991; Boulanouar, 1986; Yacoubi, 1990; Boutin, 1993a, b) et peuplent les eaux souterraines de nombreuses régions. Les Asellides se rencontrent plus rarement (Boulanouar et al., 1989). Les Microcerberides ne comportent qu'une ou deux espèces (à confirmer) et offrent une distribution restreinte (Boulanouar, 1986; Boutin, 1985; Yacoubi, 1990), tandis que les Microparasellides du genre Microcharon présentent une large répartition dans l'ensemble du Maroc et sont relativement bien diversifiés. En effet, ce dernier genre se récolte régulièrement dans les nappes phréatiques de nombreux cours d'eau. Il a été signalé précédemment dans les eaux souterraines littorales sur la côte nord marocaine (Coineau, 1971, 1982, 1985), puis dans les aquifères continentaux par Pesce et al. (1981) près de Casablanca, dans les sources du Haouz par Messouli (1984), puis par Boulanouar (1986) et Boutin (1989) des puits de Marrakech et enfin par Yacoubi (1990) dans les eaux interstitielles du Haut-Atlas et du Haouz. Plusieurs espèces encore inédites proviennent de différentes régions du Maroc. L'une d'elles, assez largement répandue dans la zone centre-ouest du 
pays, est décrite ci-dessous. Son origine et son histoire évolutive sont ensuite envisagées en relation avec la paléogéographie.

\section{Microcharon boutini n. sp.}

Matériel d'étude. - Dix-huit individus mâles et femelles dont une ovigère capturés au filet phréatobiologique, puits creusé au piémont du versant nord des Jbilet à Sidi Bouatmane (Boulanouar coll.); profondeur $30 \mathrm{~m}$, hauteur d'eau $16 \mathrm{~m}$, pH 7,2, conductivité $4,5 \mu \mathrm{S} / \mathrm{cm}^{-1}$, température $22^{\circ} \mathrm{C}$. Quelques exemplaires d'un puits de Boukricha, dans le massif des Jbilet (Boulanouar coll.). Quelques individus de plusieurs puits des vallées du Zat et de l'Ourika (Yacoubi coll.).

La série syntype comprend 30 spécimens des deux sexes dont 24 exemplaires restent dans la collection des auteurs à Marrakech et à Banyuls, le reste des individus étudiés sera déposé au Muséum National d'Histoire Naturelle de Paris et à l'Institut Scientifique de Rabat.

Derivatio nominis. - Nous dédions amicalement cette espèce à Claude Boutin qui a initié les recherches en hydrobiologie souterraine actuellement poursuivies au Maroc.

Description. - La longeur du corps varie de 1,05 à $1,3 \mathrm{~mm}$.

Antenne 1 (Fig. 1A): Comme chez la plupart des espèces d'eau douce, elle se compose de 6 articles (Coineau, 1968, 1985); la chétotaxie de cet appendice est conforme au canevas classique chez les espèces dulçaquicoles: l'article basal, le plus large, porte une courte soie pennée et une soie simple; le second article, aussi long que le premier, mais plus étroit, est pourvu de 5 soies ( 2 courtes et simples et 3 pennées) et d'une forte et longue tige pennée distale dont l'extrémité atteint presque la moitié du $5 e$ article; $3 \mathrm{e}$ article glabre; le $4 \mathrm{e}$ porte 1 soie simple proximale et 2 soies pennées distales de taille inégale, la soie extérieure étant plus longue que la soie intérieure; 5e article avec 1 courte soie simple distale et 1 batonnet hyalin relativement long chez le mâle et chez la femelle; article terminal offrant 3 soies simples (1 longue et 2 moyennes), 1 tige pennée subdistale et 1 long aesthétasque.

Antenne 2 (Fig. 1B): Le fouet et une partie de la hampe sont amputés chez tous les individus par suite de la fragilité de cet appendice et de la mé- thode de prélèvement non destinée aux études morphologiques. Premier article de la hampe court avec 1 soie simple subdistale; article 2 glabre; article 3 plus long, portant 1 soie simple distale et l'exopodite caractéristique du genre muni de 2 soies simples inégales, dont la plus apicale est la plus longue.

Labre au contour arrondi et régulier, glabre; labium muni de 2 lobes aux nombreuses soies marginales.

Mandibule (Fig. 2D, E): le processus incisif comprend 4 dents principales; cette série de 4 dents se poursuit par une petite indentation inférieure supplémentaire, présente à droite et à gauche et qui pourrait être interprétée comme une 5e dent minuscule moins bien individualisée que les 4 fortes dents distales; lacinia mobilis de la mandibule gauche tétradentée; processus molaire conique terminé par 3 longues soies pectinées; entre les pars incisiva et molaris de la mandibule droite s'implantent 3 tiges larges et festonnées, suivies de 2 soies simples, puis de 2 tiges pectinées; à gauche, on compte 2 tiges larges festonnées suivies de 2 soies courtes, puis les 2 tiges pectinées. Palpe triarticulé: articles 1 glabre; 2 muni de 2 fortes épines qui se croisent; 3 armé de 4 épines, la plus apicale étant la plus longue, les autres de plus en plus courtes vers la zone proximale de l'article; quelques rangées de cils fins visibles à la base des épines des 2 articles terminaux.

Maxille 1 (Fig. 2B): Endite interne étroit pourvu de 2 épines apicales entourées de quelques soies insérées sur les bords externe et interne; endite externe équipé de 12 épines apicales dont les 3 plus externes sont pectinées, d'une tige médiane ciliée à son extrémité, les autres épines restant lisses; bord externe de cet endite orné d'une dizaine de fines soies.

Maxille 2 (Fig. 2A): Endite interne le plus long portant 6 soies simples distales et 1 soie subdistale; bord interne garni de fines soies dont 2 longues à la base; marge externe avec quelques fines soies; endite médian avec 4 soies apicales pectinées longues et fortes; endite externe possédant 3 ou 4 soies également pectinées.

Maxillipède (Fig. 2F): Structure classique: l'épipodite atteint le bord distal du ler article du palpe; l'endite dépasse légèrement le $2 e$ article du palpe; il porte 2 rétinacles du côté interne, de nombreuses soies à sa marge interne et sur sa surface, ainsi que 


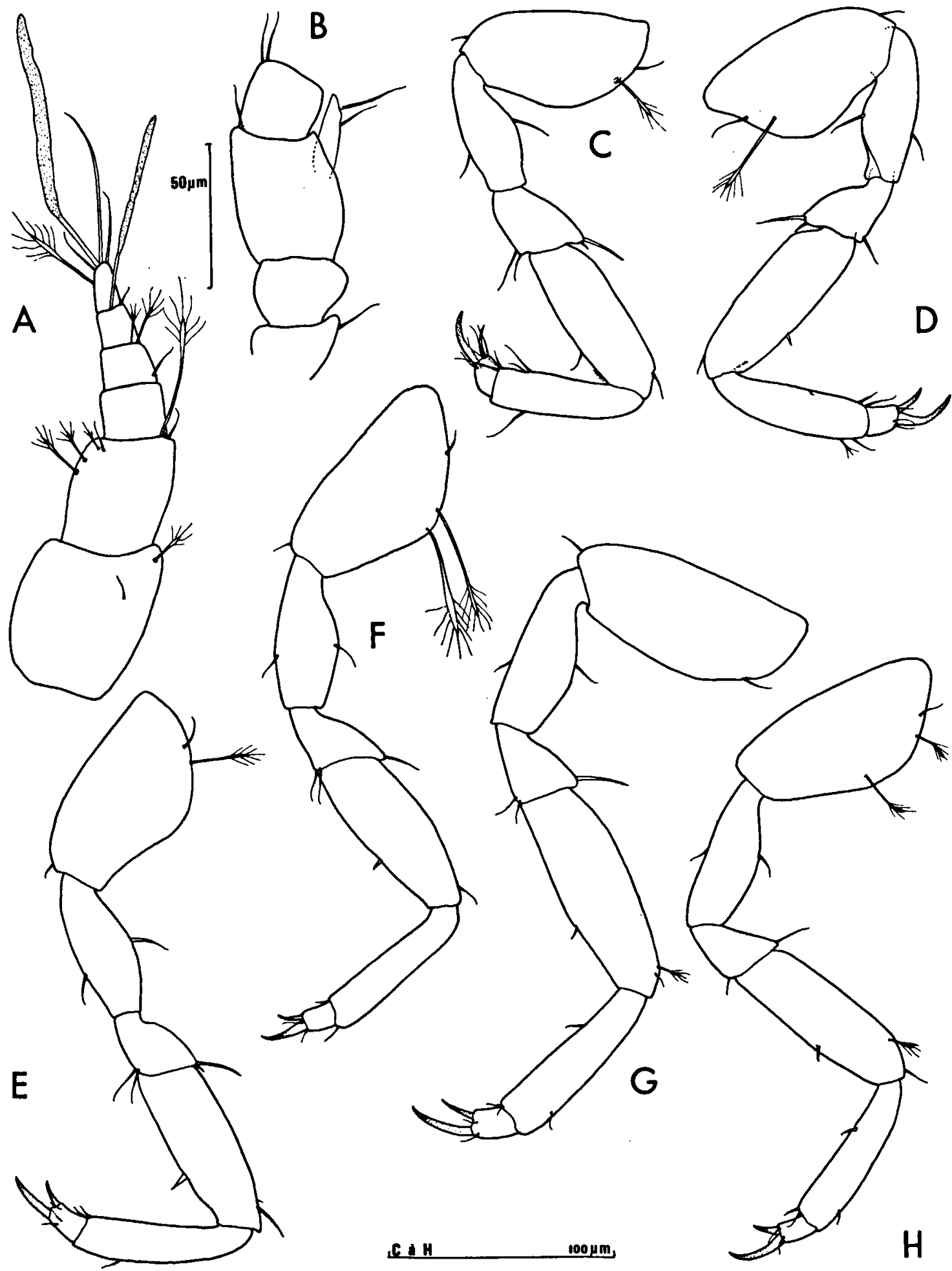

Fig. I. Microcharon boutini n. sp.: A, antenne 1; B, premiers articles de l'antenne 2; C, D, E, F, G, H, péréiopodes 1, 2, 3, 4,7 et 6. 
M. Boulanouar et al. - Un nouveau Microcharon du Maroc
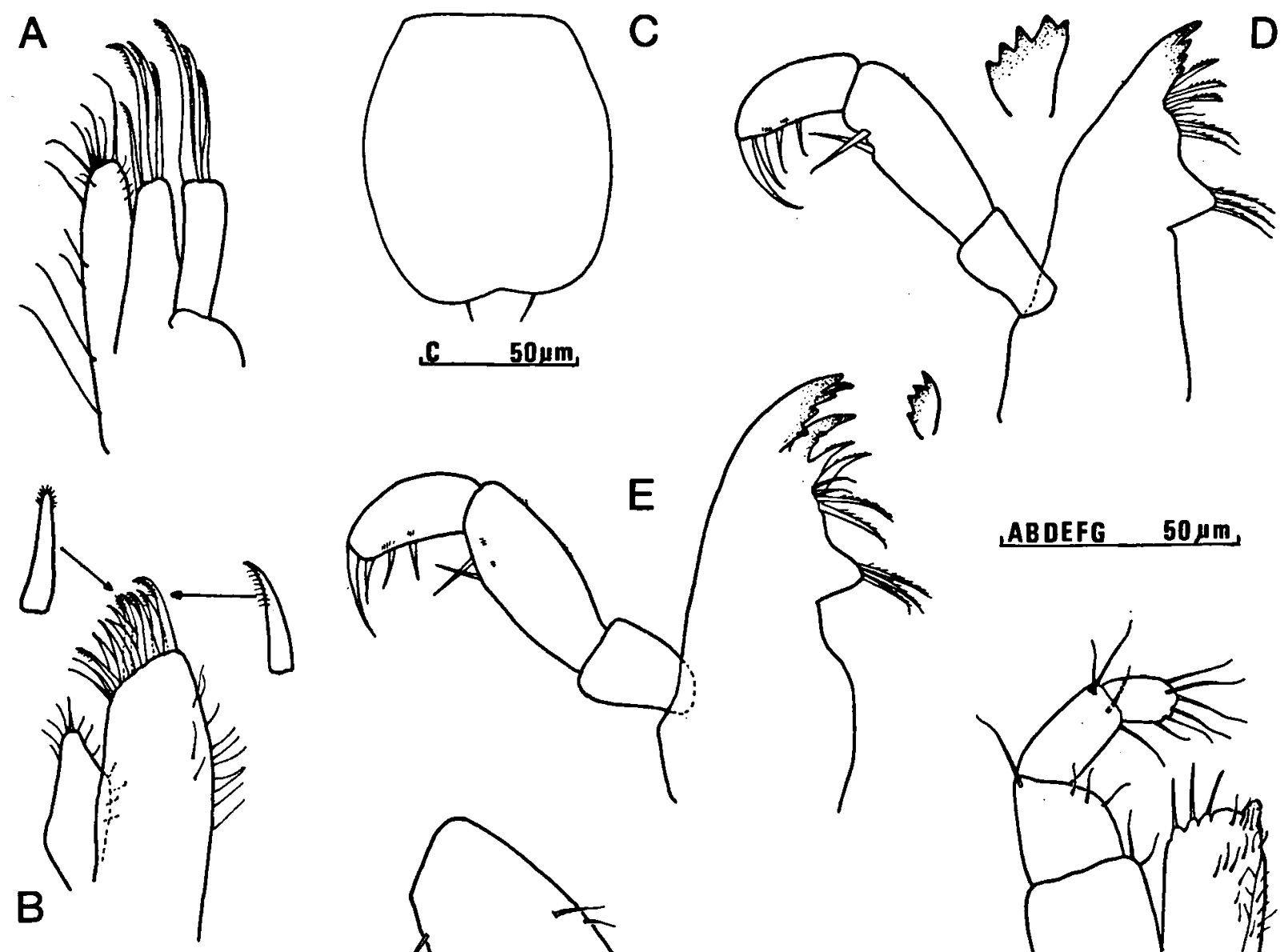


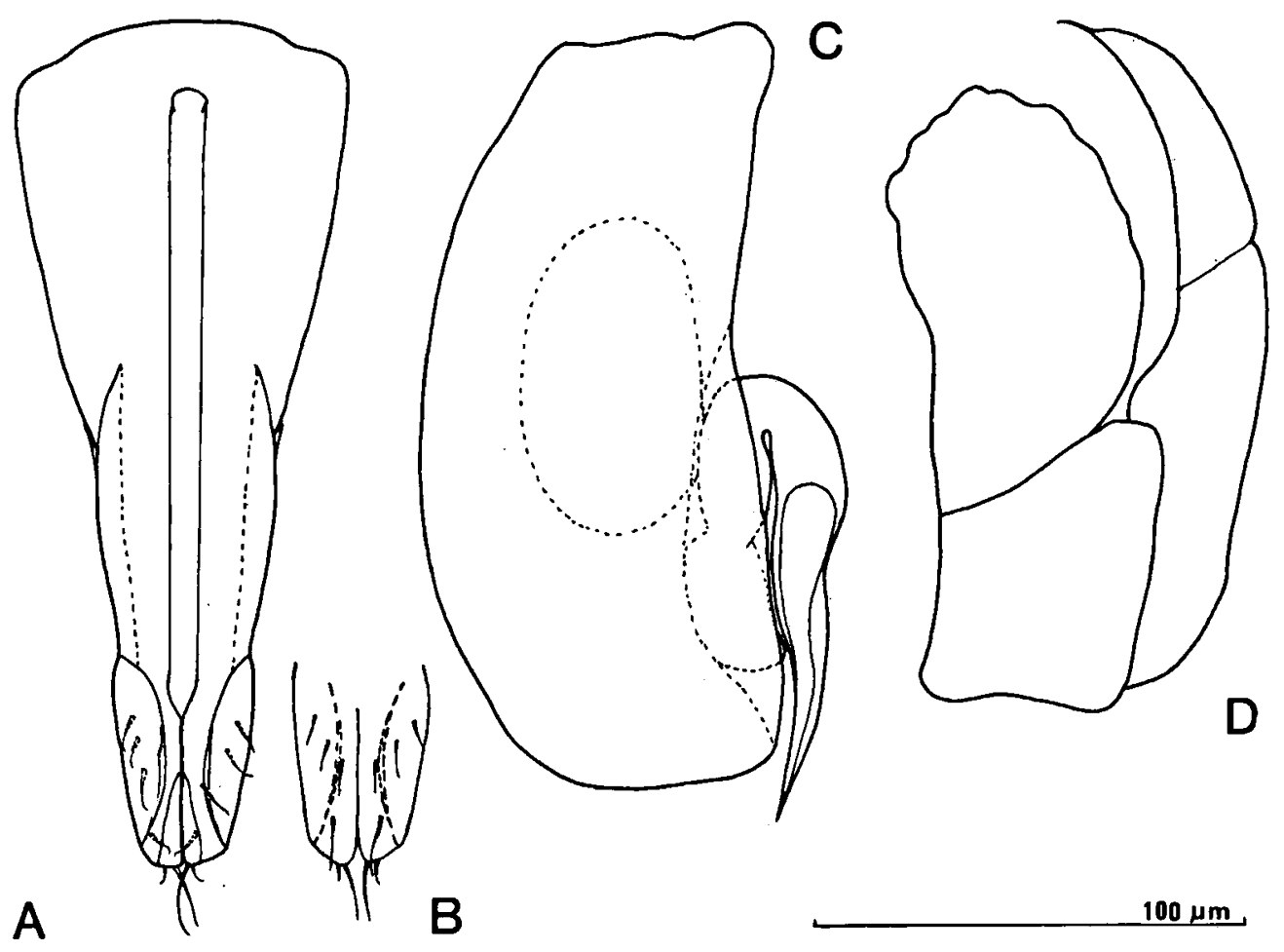

Fig. 3. Microcharon boutini n. sp.: A, pléopode 1 du mâle de la région des Jbilet; B, extrémité du pléopode 1 du mâle du piémont du Haut-Atlas de Marrakech; C, pléopode 2 du mâle; D, pléopode 3.

3 fortes soies apicales et une proéminence distalointerne ornée de quelques soies raides et courtes; palpe de 5 articles: ler article très large avec 1 soie, structure et chétotaxie des articles suivants conformes au schéma commun de la plupart des espèces du genre.

Péréiopodes (Fig. 1C-H): Longs et grêles; 1 soie simple et 1 pennée plus ou moins longue s'insèrent sur le basis relativement large de P1; le basis porte 2 soies pennées de $\mathrm{P} 4$ à P6, celui de $\mathrm{P} 7$ une seule soie lisse; les dactyles de tous les péréiopodes sont armés de 2 griffes inégales dont une relativement longue et de quelques soies subterminales.

Pléotelson (Fig. 2G): Forme rectangulaire; largeur égale aux 2/3 de la longueur et ne dépassant pas celle des segments du péréion; marge distale arrondie pourvue de 6 soies simples et de 2 tiges pennées; bords latéraux ornés de 6 soies; 6 soies dorsales.

Pléopodes 1 ơ (Fig. 3A, B): La terminologie de Cvetkov (1968) est appliquée pour décrire ces appendices. Relativement longs et coalescents dans la partie proximale élargie. Zone médiane légèrement renflée. La partie distale non soudée correspond aux exopodites selon Cvetkov, 1968. Ces exopodites présentent un renflement accentué subterminal qui individualise bien le "replis", ce dernier se terminant, ainsi que son "rebord hyalin", légèrement avant l'angle distal externe; zone distale des exopodites oblique, portant 2 soies apicales (1 longue et 1 courte), la région du "lobe interne" (Cvetkov, 1968) étant la plus développée; 1 soie subdistale très longue et relativement éloignée de la marge apicale; 3 soies plus ou moins alignées dans les sens sagittal, s'insèrent dans la zone médiane du replis, dans le dernier quart de l'appendice.

Pléopodes 2 ơ (Fig. 3C): Sympodite sub-rectangulaire, bord interne légèrement concave, bord externe convexe; exopodite court formant un petit lobe arrondi, endopodite recourbé sur lui-même, parcouru par une gouttière se terminant par l'appendix masculina en pointe effilée qui dépasse un peu le sympodite. 
Pléopode 2 \% (Fig. 2C): Sensiblement plus long que large; marge distale concave avec 2 fines et courtes soies.

Pléopodes 3 (Fig. 3D): Endopodite volumineux; exopodite biarticulé, l'article distal se termine par une courte soie arquée dépassant l'extrémité de l'endopodite.

Uropodes (Fig. 2H): Cet appendice particulièrement fragile n'a pu être observé que sur un seul individu. Sympode moins de 3 fois plus long que large aux soies peu allongées. L'exopodite très court s'insère en position subterminale; sa longueur atteint un peu moins du quart de la hauteur du sympodite; il se termine par les 3 soies terminales et subterminale usuelles; l'endopodite, relativement long, mais nettement plus court que le sympode, correspond à environ 2 fois la longueur de l'exopodite. Sa chétotaxie comprend 4 soies submédianes dont 2 pennées courtes, puis 3 soies pennées longues et 4 soies terminales.

Aucun caractère sexuel secondaire n'a été décelé chez cette espèce.

Tous les exemplaires examinés, qu'ils proviennent de Sidi Bouatmane ou des vallées du HautAtlas présentent la même morphologie, notamment en ce qui concerne les pléopodes 1 et 2 du mâle et 2 de la femelle. Tout au plus peut-on déceler un lobe interne du pléopode 1 du mâle légèrement plus allongé chez les individus du Zat et de l'Ourika, ce qui entraîne la soie subdistale un peu plus loin de la marge que chez ceux des Jbilet. Signalons aussi le renflement subterminal du bord externe des exopodites du pléopode 1 mâle légèrement moins accusé pour les exemplaires de l'Atlas.

\section{Discussion - Remarques phylogénétiques}

L'originalité de l'espèce décrite réside principalement dans les caractéristiques de la pars incisiva mandibulaire et des pléopodes sexuels du mâle. En effet, le processus incisif des deux mandibules se distingue de celui des autres espèces par la petite dent surnuméraire de la région proximale interne. En dehors des espèces incomplètement décrites connues jusqu'à ce jour, toutes les autres possèdent une pars incisiva tétradentée, à l'exception de $M$. an- tonellae Galassi, 1991 de Grèce. Dans ce dernier cas, les 5 dents sont très nettement individualisées et offrent une taille comparable, alors que chez $M$. boutini, la 5 e dent provient de la région basale de la partie incisive.

Le pléopode 1 du mâle individualise bien $M$. boutini par rapport aux espèces déjà connues du Maghreb, M. karamani et $M$. zibani (voir Pesce \& Tetè, 1978). Les 3 soies situées au niveau du quart distal de chaque exopodite s'implantent selon un alignement longitudinal, alors que leurs points d'insertion sont relativement plus groupés et forment généralement plutôt un triangle ou une ligne nettement plus oblique se situant à l'intérieur de la zone du replis chez les espèces déjà connues; dans la région apicale, l'une des 2 soies terminales est longue, l'autre courte, et il existe une soie en position subterminale de taille moyenne qui dénote un léger allongement de la région distale du lobe interne de l'exopodite dans le sens sagittal car elle se trouve plus éloignée de la marge distale que chez les espèces déjà connues; cet étirement est un peu plus marqué dans les vallées du Zat et de l'Ourika de sorte que la soie subterminale se localise un peu plus loin encore de la marge apicale (Fig. 3B). La soie 4 (ou bien la soie 6?) est absente, ce qui correspond à un caractère apomorphe. Il est à remarquer que si c'est bien la soie 6 qui manque, cette absence pourrait représenter l'indice d'un développement arrêté prématurément chez l'ancêtre de cette espèce et donc une origine progénétique. En effet, ce sont les soies 6 et 2 qui apparaissent les dernières au cours du développement post-embryonnaire (Coineau, 1971). Le bord latéral externe de l'exopodite marque un net renflement au niveau du quart terminal qui individualise la zone du "repli": ce renflement caractérise bien la nouvelle espèce. Le pléopode $1 \mathrm{du}$ mâle distingue donc clairement l'espèce marocaine des deux espèces algériennes.

Il en est de même du pléopode 2 du mâle dont le sympode s'inscrit dans un rectangle et dont l'appendix masculina dépasse nettement l'extrémité du sympode alors que l'inverse s'observe chez $\boldsymbol{M}$. karamani et $M$. zibani (voir Pesce \& Tetè, 1978).

$M$. boutini se remarque également par la tige pennée et pédonculée terminale du second article de l'antenne 1 qui reste courte contrairement à celle 
des formes d'Algérie; les autres soies pennées de cet appendice sont également courtes. L'exopodite des antennes 2 est relativement long, avec des soies un peu décalées vers la zone médiane, alors qu'elles sont généralement subdistales. Les articles 2 et 3 du palpe du maxillipède ne présentent pas un fort élargissement comme c'est souvent le cas: cet élargissement reste modéré.

La maxille 2 ne possède pas de soie pectinée distale sur l'endite interne, alors que cette dernière existe chez les deux formes algériennes.

Par ses caractères, et principalement en raison de l'allongement du lobe interne du pléopode $1 \mathrm{du}$ mâle, $M$. boutini appartient au groupe messoulii qui réunit des espèces ayant conservé des caractères primitifs (Coineau et al., sous presse); elle représente l'espèce aux caractères les plus apomorphes, constituant l'espèce-soeur de l'ensemble des autres espèces de ce groupe.

\section{Répartition, origine et biogéographie historique}

Microcharon boutini $\mathrm{n}$. sp. est présent au piémont du versant nord du massif des Jbilet ainsi que sur le massif, $8 \mathrm{~km}$ plus au sud. Il abonde aussi dans les vallées de l'Ourika et du Zat, sur les premiers contreforts du versant nord du Haut-Atlas (Figs. 4, 5).

Les événements paléogéographiques qui ont participé à l'histoire de ces régions et qui pourraient être responsables de divergences évolutives par vicariance permettent de formuler des hypothèses expliquant l'origine de $M$. boutini et sa répartition. En effet, le genre Microcharon provient d'ancêtres marins (Coineau, 1986, 1992). Diverses espèces actuelles vivent d'ailleurs encore dans les sables littoraux et marins peu profonds. Les ancêtres des espèces vivant actuellement dans les eaux souterraines continentales se seraient établis dans les eaux douces souterraines interstitielles au cours des régressions marines qui se sont produites à diverses époques géologiques. Le modèle biphase de colonisation et d'évolution (Coineau, 1985; Boutin \& Coineau, 1990; Notenboom, 1991; Coineau \& Boutin, 1992) incluant le "Regression model evolution" (Stock, 1977, 1980) détaille les deux étapes suivies par les ancêtres marins épibenthiques pour devenir des organismes interstitiels dulcicoles.

La connaissance de différentes espèces du genre Microcharon présentes au Maroc a permis d'établir les relations phylogénétiques entre ces taxons (Yacoubi Khebiza, 1990; Coineau et al., en préparation).

En ce qui concerne les espèces du groupe monophylétique auquel appartient $M$. boutini, la première phase, c'est-à-dire la pénétration active de l'ancêtre marin commun de surface dans les sables littoraux, aurait pu s'accomplir au Mésozoïque, dès la fin du Crétacé inférieur, ou même avant. Après les larges transgressions du Cénomano-Turonien, les derniers golfes marins qui ont couvert les régions occupées par $M$. boutini datent de l'Eocène, aussi bien dans la zone située au nord des Jbilet que dans celle qui est à l'emplacement actuel du Haut-Atlas (Choubert \& Faure-Muret, 1962; Trappe, 1991, 1992; Salvan, 1986; cf. Figs. 4, 5). Des populations déjà interstitielles et vivant sur les rives de ces golfes éocènes, ont pu demeurer sur place et devenir progressivement et passivement dulçaquicoles souterraines lors de l'une des régressions de la mer éocène selon le processus du "Regression model evolution" décrit par Stock (1980), aboutissant ainsi aux populations ancestrales de $M$. boutini. C'est la seconde phase ou transition horizontale du "modèle biphase". Une spéciation par vicariance se produit au terme de la régression lorsque le flux génétique entre les populations littorales qui ont accompagné le retrait marin, et les populations du nouveau stygobionte des eaux souterraines douces (ou limnostygobionte) restées sur place, est interrompu. Un tel scénario paléobiogéographique reconstituant l'origine, la colonisation des eaux souterraines continentales, l'histoire évolutive et la biogéographie historique a déjà été envisagé pour des espèces de Microcharon de France (Dole \& Coineau, 1987), d'Italie (Coineau, 1992) et de l'ensemble du Bassin méditerranéen (Coineau, 1994). La colonisation de toutes ces espèces des eaux souterraines continentales par les ancêtres littoraux s'échelonne du Turonien au Pliocène. Il en est de même au Maroc pour les diverses espèces provenant de plusieurs régions du pays. D'autres groupes de 


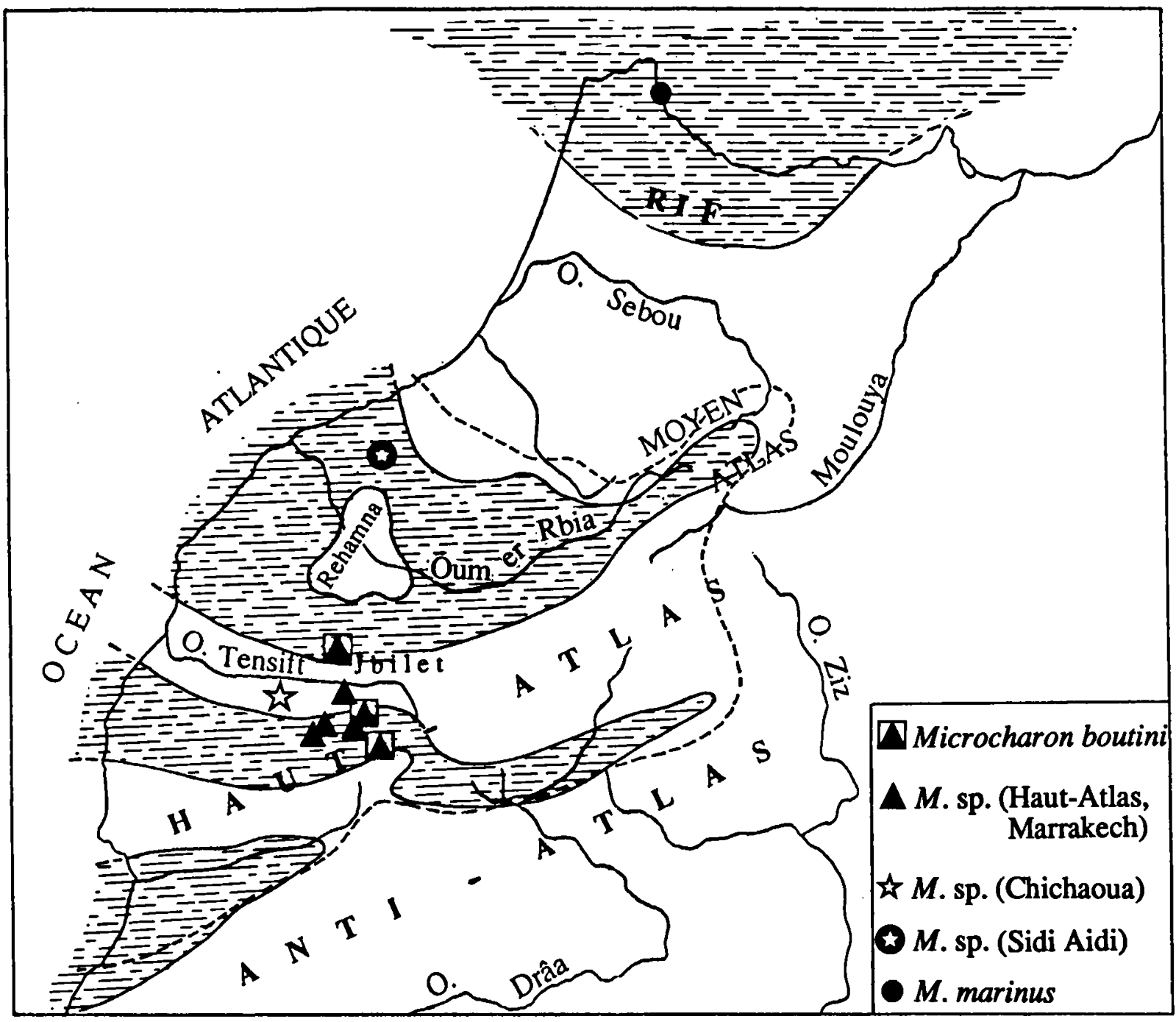

Fig. 4. Carte des régions septentrionale, centrale et occidentale du Maroc montrant la répartition des espèces du genre Microcharon et l'extension des golfes marins à l'Eocène, d'après Choubert \& Faure-Muret (1962), Salvan (1986) et Trappe (1991), modifiés.

Crustacés interstitiels stygobies continentaux répondent à un scénario biogéographique semblable. Ainsi, l'origine et la mise en place des ancêtres des Amphiphodes Pseudoniphargides du Nord marocain proviendraient de la régression de la fin du Tortonien dans le sillon péri-rifain (Coineau \& Boutin, 1986; Boutin \& Coineau, 1988). Pour les Amphipodes Metacrangonyctidae et les Isopodes Cirolanidae, les ancêtres stygobies interstitiels marins des divers groupes d'espèces se sont introduits dans les eaux douces lors des régressions turonnienne, sénonienne et éocène (Boutin et al., 1992; Boutin, 1993a, b; Boutin, 1994; Messouli, 1994).
Si le piémont des Jbilet a bien été submergé par la transgression éocène, la zone intérieure de ce massif située au sud de Sidi Bouatmane qui recèle $M$. boutini, ainsi que d'autres taxons d'origine marine comme des Thermosbaenacés, pose une énigme si l'on considère son histoire paléogéographique telle qu'elle est connue par la littérature. En effet, on considère en général que le massif primaire des Jbilet, comme celui des Rehamna, serait resté émergé lors des transgressions successives du Cénomano-Turonien, du Maastrichtien et de l'Eocène (Choubert et Faure-Muret, 1962; Michard, 1976). Cependant, Trappe (1991) indique que "The out- 


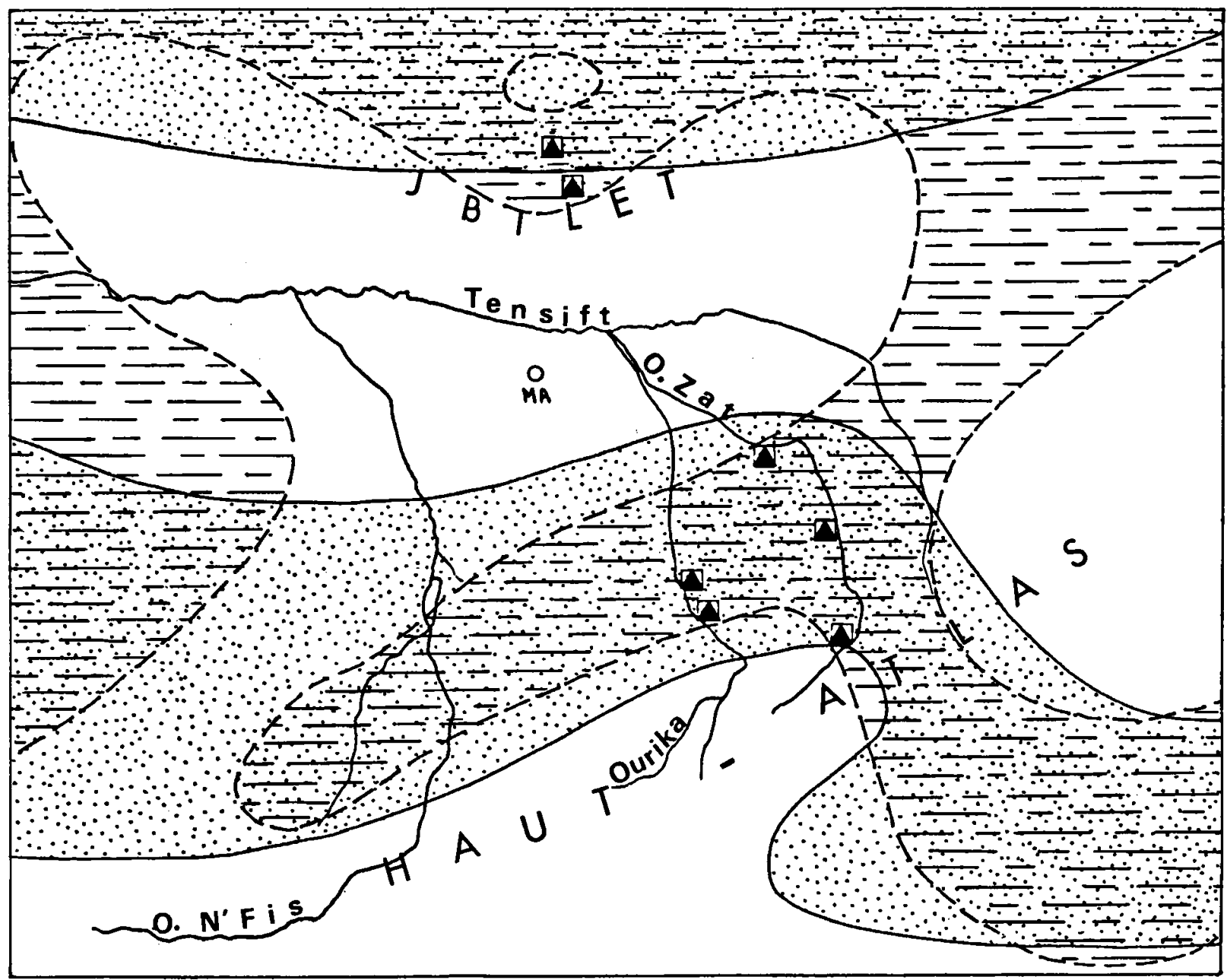

Fig. 5. Carte de la région des Jbilet, du Haouz et du versant nord du Haut-Atlas de Marrakech, indiquant la répartition de Microcharon boutini $\mathrm{n}$. sp. et l'extension des golfes éocènes (zones en pointillés) selon Choubert \& Faure-Muret (1962); zones hachurées horizontalement selon Salvan (1986). (MA = Marrakech.)

crops of Paleozoic basement (Jebilet, Rehamna) could not be proved as continental areas. They are, at best, swells". Il ne paraît donc pas impossible que ces vallonnements ou ondulations de faible altitude puissent correspondre à une série d'îles à l'emplacement des Jbilet à l'époque de la mer des phosphates et qu'un bras de mer ait pu occuper la vallée actuelle de l'oued Boukricha. Cette hypothèse pourrait bien expliquer la présence de $M$. boutini ainsi que celle des Monodelles dans les eaux souterraines de Boukricha, à l'intérieur des Jbilet.

Une migration active de l'ancêtre de l'espèce dé- posé par le golfe éocène au piémont des Jbilet pourrait-elle être envisageable, vue la faible distance $(8 \mathrm{~km})$ qui sépare l'oued Boukricha de Sidi Bouatmane? Dans ce cas, si l'on tient compte des réseaux hydrographiques actuels, cette migration se serait effectuée en sens inverse du courant souterrain. Mais le sens de l'écoulement des eaux aurait pu s'inverser au Post-Lutétien par exemple, ou à l'Oligocène lors d'une surélévation du massif des Jbilet en réponse aux deux phases d'orogenèse alors très actives de l'Atlas. De nouveaux réseaux hydrographiques ont pu se mettre en place, des réseaux 
anciens ont aussi pu se modifier lors de chacune de ces phases orogéniques de l'Atlas comme ce fut le cas par exemple dans l'Anti-Atlas, après l'installation dans les eaux souterraines de l'ancêtre de $M$. boutini. Cependant, rappelons aussi que les minuscules Isopodes interstitiels, comme les autres stygobiontes interstitiels, n'ont que de très faibles capacités migratoires, en l'absence de larves nageuses, du fait de leur développement holobenthique. D'autre part, ils sont inféodés aux biotopes sableux en raison d'un thigmotactisme puissant, de sorte qu'une migration active suppose la continuité géologique des strates sableuses souterraines.

Nous penchons donc plutôt pour la première hypothèse faisant appel à la submersion par la mer éocène des zones les plus basses des Jbilet (Trappe, 1991).

Par ailleurs, la présence de Microcharon boutini n. sp. à la fois dans la zone des Jbilet et dans celle du Haut-Atlas pourrait s'interpréter comme un témoignage de la lenteur relative de l'évolution morphologique dans les milieux interstitiels continentaux au sein du genre Microcharon. En effet, les populations ancestrales de cette espèce sont parvenues dans les eaux douces souterraines au plus tard à la fin de l'Eocène. On ne constate que de très faibles différences entre les individus de ces deux régions, qui ressortent du domaine des variations géographiques existant généralement au sein d'une même espèce. Cette constatation contraste cependant avec l'endémisme marqué que l'on peut remarquer pour différentes espèces du genre appartenant au même groupe phylogénétique et qui occupent actuellement les vallées du Haut-Atlas central (Yacoubi Khebiza, 1990; Coineau et al., en préparation); l'une de ces dernières vit dans la vallée de l'Ourika et cohabite avec $M$. boutini dans deux stations.

Il n'est pas impossible de considérer que les différences ténues qui distinguent les populations de $M$. boutini de l'Ourika et du Zat de celles des Jbilet, correspondent aux premiers stades d'une spéciation par vicariance en cours de réalisation, du fait de l'isolement prolongé des populations des Jbilet et du piémont de l'Atlas. Par contre, la présence de synapomorphies bien caractérisées chez les populations de $M$. boutini révèle une évolution plus importante de l'ancêtre commun encore littoral (thalassostygobionte) lorsqu'il vivait dans l'interstitiel des golfes éocènes. Cette différence d'amplitude des variations morphologiques dans les deux types de milieux interstitiels suggère que chez les Isopodes Microparasellidae du genre Microcharon, comme chez d'autres groupes de stygobiontes thalassoïdes tels que les Amphipodes Metacrangonyctidae et les Isopodes Cirolanidae, l'évolution a été plus rapide dans les milieux littoraux instables que dans les biotopes continentaux relativement plus stables (Boutin \& Coineau, 1991; Boutin, 1994; Messouli, 1994).

\section{Conclusion}

Microcharon boutini $\mathrm{n}$. sp. représente la première d'une série d'espèces inédites découvertes récemment au Maroc. Ses caractères originaux la distinguent nettement des deux autres espèces continentales connues d'Algérie. Elle se rencontre au pied des Jbilet, à l'intérieur de ce massif, ainsi qu'au piémont du Haut-Atlas de Marrakech. L'établissement des populations ancestrales marines dans les eaux douces souterraines remonte à la régression de la mer des phosphates de l'Eocène.

\section{Remerciements}

Ces travaux se poursuivent dans le cadre de l'Action intégrée Franco-Marocaine $n^{\circ}$ 93/643 et du GDR 88 “Evolution des organismes microscopiques". Nous remercions C. Boutin pour ses critiques constructives et ses suggestions lors de la lecture d'une première version de ce manuscrit.

\section{Bibliographie}

Boulal, M., 1988. Recherches écologiques sur la faune aquatique des puits de la région de Tiznit (Anti-Atlas occidental, Maroc): 1-228 (Thèse 3e cycle, Univ. Marrakech).

Botosaneanu, L., C. Boutin \& J.-P. Henry, 1985. Deux remarquables Cirolanides stygobies nouveaux du Maroc et de Rhodes. Stygologia, 1 (2): 186-207.

Boulanouar, M., 1986. Etude écologique comparée de quelques puits de la région de Marrakech. Impact des pollutions sur la zoocénose des puits: 1-159 (Thèse 3e cycle, Univ. Marrakech). 
Boulanouar, M., J.-P. Henry \& C. Boutin, 1989. Premier Aselle anophtalme du Maroc: Proasellus gineti n. sp. (Crustacea, Isopoda, Asellota). Stygologia, 6 (2): 119-125.

Boutin, C., 1985. Sensibilité à la pollution et répartition de quelques espèces de Crustacés phréatobies à Marrakech (Maroc occidental). Mém. Biospéol., 11 “1984”: 55-64.

Boutin, C., 1989. Processes and chronology of the transition towards continental groundwaters of the stygobiontic Isopoda (Crustacea) of Morocco. Comm. 7th Internat. Meiofauna Conf., Vienna, August 1989.

Boutin, C., 1993a. Biogéographie historique des Crustacés Malacostracés stygobies du Maroc: 1-262 (Thèse Doct., Univers. Lyon I).

Boutin, C., 1993b. Biogéographie historique des Crustacés Isopodes Cirolanidae stygobies du groupe Typhlocirolana dans le bassin méditerranéen. C. R. hebd. Séanc. Acad. Sci. Paris, Sci. Vie, 316 (12): 1505-1510.

Boutin, C., 1994. Biogeography of the metacrangonyctid amphipods in North Africa. Hydrobiologia, 287 (1): 49-64.

Boutin, C. \& M. Boulanouar, 1984. Premières données sur la faune des puits de Marrakech. Verh. int. Verein. Limnol., 22: 1762-1765.

Boutin, C. \& M. Boulanouar, 1991. Découverte d'un nouveau genre de Crustacé Isopode Cirolanide dans les eaux souterraines continentales du Maroc. Nouvelles hypothèses sur la biogéographie historique des Cirolanides du Bassin méditerranéen. Comm. Congr. Int. Biospéol., 9-13 sept 1991, Liège: 37.

Boutin, C. \& N. Coineau, 1988. Pseudoniphargus maroccanus n. sp. (subterranean amphipod), the first representative of the genus in Morocco. Phylogenetic relationships and palaeobiogeography. Crustaceana, Suppl. 13: 1-19.

Boutin, C. \& N. Coineau, 1990: "Regression Model”, "Modèle biphase" d'évolution et origine des micro-organismes stygobies interstitiels continentaux. Revue Micropaléont., 33 (3/4): 303-332.

Boutin, C. \& N. Coineau, 1991. Instabilité des conditions environnementales et vitesse de l'évolution. L'exemple des micro-Crustacés souterrains d'origine marine dans les pays méditerranéens. Bull. Inst. Géol. Bassin Aquitaine, 50: 6369.

Boutin, C., M. Messouli \& N. Coineau, 1992. Phylogénie et biogéographie évolutive d'un groupe de Metacrangonyctidae, Crustacés Amphipodes stygobies du Maroc. II. Cladistique et paléobiogéographie. Avec l'examen comparatif de plusieurs logiciels de parcimonie. Stygologia, 7 (3): 159-177.

Choubert, G. \& A. Faure-Muret, 1962. Evolution du domaine atlasique marocain depuis les temps paléozoïques. In: Livre à la mémoire du Professeur Paul Fallot, 1: 447-527 (Mém. h.s. Soc. géol. France, Paris).

Coineau, N., 1968. Contribution à l'étude de la faune interstitielle. Isopodes et Amphipodes. Mém. Mus. natn. Hist. nat., N.S., Sér. A, Zool., 55 (3): 147-216.

Coineau, N., 1971. Les Isopodes interstitiels. Documents sur leur écologie et leur biologie. Mém. Mus. natn. Hist. nat., N.S., Sér. A, 64: 1-170.
Coineau, N., 1982. Microcharon Kar. et Angeliera Chap. et Delam., Crustacés Isopodes Asellotes interstitiels Microparasellides. Documents pour un atlas zoogéographique du Languedoc-Roussillon, 24: 1-4.

Coineau, N., 1985. Colonisation du milieu souterrain continental par les organismes d'origine marine. Moyen d'accès par le milieu interstitiel. Bull. Soc. Biospéol., 8: 26-30.

Coineau, N., 1986. Isopoda: Asellota: Janiroidea. In: L. Botosaneanu (ed.), Stygofauna mundi: 465-472 (E.J. Brill/ W. Backhuys, Leiden).

Coineau, N., 1992. Biogéographie évolutive du Crustacé interstitiel Microcharon (Isopoda, Janiroidea) dans l'ouest du Bassin Méditerranéen. Bull. Inst. océanogr., Monaco, 9: 101114.

Coineau, N., 1994. Evolutionary biogeography of the microparasellid isopod Microcharon (Crustacea) in the Mediterranean Basin. In: Biogeography of subterranean crustaceans: the effects of different scales. Summer Meeting of the Crustacean Society, Charleston, June 1992. Hydrobiologia, 287 (1): 79-96.

Coineau, N. \& C. Boutin, 1986. Le genre Pseudoniphargus (Crustacea, Amphipoda) au Maroc. Paléobiogéographie. Boll. Zool., 53 (Suppl.): 102.

Coineau, N. \& C. Boutin, 1992. Biological processes in space and time. Colonization, evolution and speciation in interstitial stygobionts. In: A.I. Camacho (ed.), The natural history of biospeleology. Mus. nac. Cie. nat., CSIC Ed., Madrid, Monografías, 7: 423-451.

Cvetkov, L., 1968. Morphologie des premiers pléopodes, évolution et position systématique du genre Microcharon (Crustacea, Isopoda). Izv. zool. Inst. Sof., 27: 107-140.

Dole, M.-J. \& N. Coineau, 1987. L'Isopode Microcharon (Crustacea, Isopoda) abondant dans les eaux interstitielles de l'est Lyonnais. M. reginae n. sp., écologie et biogéographie. Stygologia, 3 (3): 200-216.

Galassi, D.M.P., 1991. A new Microcharon Karaman, from hyporheic waters of Greece: Microcharon antonellae $n$. sp. (Crustacea Isopoda: Microparasellidae). Stygologia 6 (4): 201-207.

Idbennacer, B., 1990. Recherches écologiques, biogéographiques et démographiques sur la faune aquatique souterraine de la région de Guelmim (Sud-Ouest de l'Anti-Atlas marocain): 1-275 (Thèse 3e cycle, Fac. Sc. Semlalia, Marrakech).

Messouli, M., 1984. Recherches de la faune stygobie des sources de la région de Marrakech. Mém. DEA, Univ. Marrakech: 1-35.

Messouli, M., 1994. Evolution, phylogénie et biogéographie historique des Metacrangonyctidae, Crustacés Amphiphodes stygobies du Nord de l'Afrique et des régions voisines: 1-309 (Thèse d'Etat, Univ. Marrakech).

Michard, A., 1976. Eléments de géologie du Maroc. Notes Mém. Serv. Mines Carte géol. Maroc, 252: 1-408.

Notenboom, J., 1991. Marine regressions and the evolution of groundwater dwelling amphipods (Crustacea). J. Biogeography, 18: 437-454.

Nourisson, M., 1956. Etude morphologique comparative et cri- 
tique des Typhlocirolana (Crustacés Isopodes Cirolanidae) du Maroc et d'Algérie: Bull. Soc. Sci. nat. phys. Maroc, 36: 103-124.

Pesce, G.L. \& P. Tetè, 1978. Recherches en Afrique de l'Institut de Zoologie de l'Aquila (Italie). I. Microparasellides d'Algérie (Crustacea: Isopoda). Rev. Zool. afr., 92 (4): 992-1002.

Pesce, G.L., P. Tetè \& P. de Simone, 1981. Ricerche faunistiche in acque sotterranee del Maghreb (Tunisia, Algeria, Marocco) e d'ell'Egitto. Natura, Milano, 72 (1-2): 63-98.

Salvan, M.H., 1986. Géologie des gîtes minéraux marocains (2e éd.), 3. Phosphates. Notes Mém. géol. Maroc, 276: 1392.

Stock, J.H., 1977. The taxonomy and zoogeography of the hadziid Amphipoda, with emphasis on the West Indian taxa. Stud. Fauna Curaçao, 55: 1-130.

Stock, J.H., 1980. Regression model evolution as exemplified by the genus Pseudoniphargus (Amphipoda). Bijdr. Dierk., 50 (1): 105-144.

Trappe, J., 1991. Stratigraphy, facies distribution and paleogeography of the marine Paleogene from the western High Atlas, Morocco. Neues Jb. Geol. Paläont. Abh., 180 (3): 279-321.

Trappe, J., 1992. Microfacies zonation and spatial evolution of a carbonate ramp: marginal Moroccan phosphate sea during the Paleogene. Geologische Rundschau, 81 (1): 105-126.

Yacoubi Khebiza, M., 1990. Ecologie et biogéographie des biocénoses aquatiques des nappes alluviales de quelques vallées du Haut-Atlas de Marrakech (Maroc). Paléobiogéographie des Crustacés phréatobies: 1-246, annexes 1-9 (Thèse 3e cycle, Univ. Marrakech).

Reçu le 26 juillet 1994 